\title{
A gap theorem for hypersurfaces of the sphere with constant scalar curvature one
}

Hilário Alencar*, Manfredo do Carmo* and Walcy Santos*

\begin{abstract}
We consider closed hypersurfaces of the sphere with scalar curvature one, prove a gap theorem for a modified second fundamental form and determine the hypersurfaces that are at the end points of the gap. As an application we characterize the closed, two-sided index one hypersurfaces with scalar curvature one in the real projective space.
\end{abstract}

Mathematics Subject Classification (2000). 53C42.

Keywords. Scalar curvature, sphere, Clifford torus, index one, projective space.

\section{Introduction}

To state our main result we need some notation.

$x: M^{n} \rightarrow S^{n+1}(1)$ will be a closed (compact without boundary) hypersurface of the unit sphere $S^{n+1}(1)$. We denote by $A$ the linear map associated to the second fundamental form and by $k_{1}, \ldots, k_{n}$ its eigenvalues (principal curvatures of $M)$. We will use the first two elementary symmetric function of the principal curvatures:

$$
S_{1}=\sum_{i=1}^{n} k_{i}, S_{2}=\sum_{i<j=1}^{n} k_{i} k_{j} .
$$

We will also use the normalized means: the mean curvature $H=\frac{1}{n} S_{1}$ and the scalar curvature $R$, given by $n(n-1)(R-1)=S_{2}$. Finally, we introduce the first two Newton tensors by

$$
P_{0}=I d, P_{1}=S_{1} I d-A .
$$

Clearly $P_{1}$ commutes with $A$ and it is also a self-adjoint operator. We will show later (see Remark 2.1) that if $R=1$ and $S_{1} \geq 0$, then all eigenvalues of $P_{1}$ are nonnegative, hence we can consider $\sqrt{P_{1}}$.

\footnotetext{
*Partially supported by CNPq, Brazil.
} 
We can now state our gap theorem.

Theorem 1. Let $x: M^{n} \rightarrow S^{n+1}(1)$ be a closed orientable hypersurface with scalar curvature $R=1$ (equivalently, $S_{2}=0$ ). Assume that $S_{1}$ does not change sign and choose the orientation such that $S_{1} \geq 0$. Assume further that

$$
\left\|\sqrt{P_{1}} A\right\|^{2} \leq \operatorname{trace} P_{1} \text {. }
$$

Then:

(i) $\left\|\sqrt{P_{1}} A\right\|^{2}=\operatorname{trace} P_{1}$.

(ii) $M^{n}$ is either a totally geodesic submanifold or $M^{n}=S^{n_{1}}\left(r_{1}\right) \times S^{n_{2}}\left(r_{2}\right) \subset$ $S^{n+1}(1)$, where $n_{1}+n_{2}=n, r_{1}^{2}+r_{2}^{2}=1$ and $\left(\frac{r_{2}}{r_{1}}\right)^{2}=\beta$ satisfies the quadratic equation:

$$
n_{1}\left(n_{1}-1\right) \beta^{2}-2 n_{1} n_{2} \beta+n_{2}\left(n_{2}-1\right)=0 .
$$

Our theorem was inspired by a similar theorem on minimal submanifolds of the sphere first proved by J. Simons [S] (part (i)) and latter completed (part (ii)) by S. S. Chern, M. do Carmo and Kobayashi [CdCK] and, independently, by H. B. Lawson [L].

Remark. The condition on the modified second fundamental form in above theorem can not be dropped, as can be seen by the following example: Let $M^{6} \rightarrow S^{7}(1)$ be an isoparametric hypersurface with principal curvatures given by

$$
\lambda_{1}=\lambda_{2}=\theta, \lambda_{3}=\frac{\theta+1}{1-\theta}, \lambda_{4}=\lambda_{5}=-\frac{1}{\theta} \text { and } \lambda_{6}=-\frac{1-\theta}{1+\theta}
$$

where $\theta$ is given by $\theta=\sqrt{\frac{13+\sqrt{165}}{2}}($ see $[\mathrm{M}])$. It is easy to see that $M^{6}$ has $R=1$ and $S_{1}>0$. We would like to thank Luiz Amancio de Sousa Junior for showing us this example.

As an application of Theorem 1, we will present a characterization of index one closed hypersurfaces with constant scalar curvature one of the real projective space $\mathbb{P}(\mathbb{R})^{n+1}$. For minimal submanifolds this result was obtained recently by $M$. do Carmo, M. Ritoré and A. Ros [dCRR].

Before giving a formal statement we need some considerations. Hypersurfaces of a curvature one space form with constant scalar curvature one are solutions to a variational problem (see $[\mathrm{Re}],[\mathrm{Ro}],[\mathrm{BC}]$ ) whose Jacobi equation is

$$
T_{1} f=L_{1} f+\left\{\left\|\sqrt{P_{1}} A\right\|^{2}+\operatorname{trace} P_{1}\right\} f=0 .
$$

Here $f \in C^{\infty}(M)$ and $L_{1}$ is a second order differential operator given by

$$
L_{1} f=\operatorname{div}\left(P_{1} \nabla f\right),
$$


where $\nabla f$ is the gradient of $f$. Notice that $L_{1}$ generalizes the Laplacian. However, differently from the Laplacian, $L_{1}$ is not always elliptic. J. Hounie and M. L. Leite [HL] have proved that if $S_{3} \neq 0$ everywhere, then $L_{1}$ is elliptic. Of course, from the definition of $L_{1}$, it follows that $L_{1}$ is elliptic if and only if $P_{1}$ is positive definite (or negative definite). For the next theorem we will assume that $L_{1}$ is elliptic and $P_{1}$ is positive definite. Denote by $\operatorname{Ind}(M)$ the Morse index of $M$, i.e., the number of negative eigenvalues of $T_{1}$.

Theorem 2. Let $x: M^{n} \rightarrow \mathbb{P}(\mathbb{R})^{n+1}(1)$ be a closed two-sided hypersurface with scalar curvature one. Then $\operatorname{Ind}(M) \geq 1$ and if $\operatorname{Ind}(M)=1, M$ is the Clifford hypersurfaces obtained by the projection of the Clifford torus of Theorem 1.

\section{Preliminaries}

In this section we will present some properties of the $r^{\text {th }}$ Newton tensors in $M$ and describe the Clifford hypersurfaces of $\mathbb{P}(\mathbb{R})^{n+1}$.

\subsection{The $r^{\text {th }}$ Newton tensors}

We introduce the $r^{\text {th }}$ Newton tensors, $P_{r}: T_{p} M \rightarrow T_{p} M$, which are defined inductively by

$$
\begin{aligned}
& P_{0}=I, \\
& P_{r}=S_{r} I-A P_{r-1}, r>1,
\end{aligned}
$$

where $S_{r}=\sum_{i_{1}<\cdots<i_{r}} k_{i_{1}} \ldots k_{i_{r}}$ is the $r^{t h}$ symmetric function of the principal curvatures $k_{1}, \ldots, k_{n}$.

It is easy to see that each $P_{r}$ commutes with $A$ and if $e_{i}$ an eigenvector of $A$ associated to principal curvature $k_{i}$, then

$$
P_{1}\left(e_{i}\right)=\mu_{i} e_{i}=\left(S_{1}-k_{i}\right) e_{i} .
$$

In [Re], Reilly showed that the $P_{r}$ 's satisfy the following

Proposition 2.1 ([Re], see also [BC] - Lemma 2.1). Let $x: M^{n} \rightarrow N^{n+1}$ be an isometric immersion between two Riemannian manifolds and let $A$ be its second fundamental form. The $r$ 'th Newton tensor $P_{r}$ associated to A satisfies:

1. $\operatorname{trace}\left(P_{r}\right)=(n-r) S_{r}$,

2. $\operatorname{trace}\left(A P_{r}\right)=(r+1) S_{r+1}$,

3. $\operatorname{trace}\left(A^{2} P_{r}\right)=S_{1} S_{r+1}-(r+2) S_{r+2}$.

It follows from (3) that if $S_{2}=0, \operatorname{trace}\left(A^{2} P_{1}\right)=-3 S_{3}$. 
Remark 2.1. Observe that if $S_{2}=0$, we have that

$$
S_{1}^{2}=|A|^{2}+2 S_{2} \geq k_{i}^{2}, \text { for all } i .
$$

Thus, $0 \leq\left(S_{1}^{2}-k_{i}^{2}\right)=\left(S_{1}-k_{i}\right)\left(S_{1}+k_{i}\right)$, what implies that all eigenvalues of $P_{1}$ are nonnegative if $S_{1} \geq 0$, that is, $P_{1}$ is a nonnegative operator. We also remark that if $S_{2}=0$ and $P_{1}$ has one eigenvalue equal to zero, then

$$
P_{1} A \equiv 0 \text {. }
$$

In fact, if $\mu_{i_{0}}=0$, then $k_{i_{0}}=S_{1}$. As $S_{1}^{2}=|A|^{2}$, we get

$$
\sum_{i \neq i_{0}} k_{i_{0}}^{2}=0
$$

So $k_{i}=0$, for all $i \neq i_{0}$, hence $P_{1} A \equiv 0$.

Associated to each Newton tensor $P_{r}$, we define a second order differential operator

$$
L_{r}(f)=\operatorname{trace}\left(P_{r} \operatorname{Hess} f\right) .
$$

If $N^{n+1}$ has constant sectional curvature, it follows from Codazzi equation (see Rosenberg [Ro], p. 225) that $L_{r}$ is

$$
L_{r}(f)=\operatorname{div}_{M}\left(P_{r} \nabla f\right) .
$$

Hence $L_{r}$ is a self-adjoint operator and for any differentiable functions $f$ and $g$ on $M^{n}$

$$
\int_{M} f L_{r} g d M=\int_{M} g L_{r} f d M
$$

We observe that for $r=0, L_{0}$ is the Laplacian which is always an elliptic operator. For $r>0$ we have to add some extra condition in order to ensure that $L_{r}$ is elliptic. For hypersurfaces of $\mathbb{R}^{n+1}$ with $S_{r}=0$, Hounie and Leite, [HL], were able to give a geometric condition that is equivalent to $L_{r}$ being elliptic. In fact their proof can be generalized to hypersurfaces of the sphere and we have that

Theorem 2.1 ([HL] - Proposition 1.5). Let $M$ be a hypersurface in $\mathbb{R}^{n+1}$ or $S^{n+1}$ with $S_{r}=0,2 \leq r<n$. Then the operator $L_{r-1}(f)=\operatorname{div}\left(P_{r-1} \nabla f\right)$ is elliptic at $p \in M$ if and only if $S_{r+1}(p) \neq 0$.

Thus, for hypersurfaces with $S_{2}=0, L_{1}$ is an elliptic operator if and only if $S_{3} \neq 0$. Since $L_{1}(f)=\operatorname{div}_{M}\left(P_{1} \nabla f\right)$, it follows that the ellipticity of $L_{1}$ implies that $P_{1}$ is definite, hence then $S_{1} \neq 0$.

Let $a \in \mathbb{R}^{n+2}$ be a fixed vector. Let $x: M \rightarrow S^{n+1}(1) \subset \mathbb{R}^{n+2}$ be an isometric immersion with $S_{2}=0$ and let $N$ be its unit normal vector. The functions $f=$ $\langle N, a\rangle$ and $g=\langle x, a\rangle$ satisfy (see [BC], lemma 5.2)

$$
L_{1}(g)=-(n-1) S_{1} g
$$


and

$$
L_{1}(f)=3 S_{3} f
$$

\subsection{Clifford hypersurfaces of $\mathbb{P}(\mathbb{R})^{n+1}$}

We are now going to describe some properties of the Clifford hypersurface in $\mathbb{P}(\mathbb{R})^{n+1}$. A Clifford torus in $S^{n+1}(1)$ is given by the product immersion of $M=S^{n_{1}}\left(r_{1}\right) \times S^{n_{2}}\left(r_{2}\right)$, with $n_{1}+n_{2}=n$ and $r_{1}^{2}+r_{2}^{2}=1$, which is a closed hypersurface of $S^{n+1}(1)$. It is easy to see that this immersion is invariant under the antipodal map, hence it induces an immersion of $M$ into $\mathbb{P}(\mathbb{R})^{n+1}$. This hypersurface will be called Clifford hypersurface. If $x: S^{n_{1}}\left(r_{1}\right) \times S^{n_{2}}\left(r_{2}\right) \rightarrow S^{n+1}(1)$ is a Clifford torus, then the unit normal vector at a point $p=\left(p_{1}, p_{2}\right) \in S^{n_{1}}\left(r_{1}\right) \times S^{n_{2}}\left(r_{2}\right)$ is given by

$$
N=\left(-\frac{r_{2}}{r_{1}} p_{1}, \frac{r_{1}}{r_{2}} p_{2}\right) .
$$

Thus, the principal curvatures of $M$ are $\frac{r_{2}}{r_{1}}$ with multiplicity $n_{1}$ and $-\frac{r_{1}}{r_{2}}$ with multiplicity $n_{2}$. It is easily checked that the scalar curvature of $M$ is equal to one $\left(S_{2}=0\right)$ if and only if $\left(\frac{r_{2}}{r_{1}}\right)^{2}=\beta$ satisfies the quadratic equation:

$$
n_{1}\left(n_{1}-1\right) \beta^{2}-2 n_{1} n_{2} \beta+n_{2}\left(n_{2}-1\right)=0 .
$$

We will show in a while that only one of the torus given by (5) yields $S_{1}>0$. Notice that $L_{1}$ is an elliptic operator and in order to calculate the index of $M$, we first observe that in a principal basis, $P_{1}$ is a diagonal matrix whose elements are

$$
\left\{\left(n_{1}-1\right) \frac{r_{2}}{r_{1}}-n_{2} \frac{r_{1}}{r_{2}}\right\} \text { with multiplicity } n_{1}
$$

and

$$
\left\{n_{1} \frac{r_{2}}{r_{1}}-\left(n_{2}-1\right) \frac{r_{1}}{r_{2}}\right\} \text { with multiplicity } n_{2} \text {. }
$$

Thus,

$$
\operatorname{trace} P_{1}=(n-1) S_{1}=(n-1)\left(n_{1} \frac{r_{2}}{r_{1}}-n_{2} \frac{r_{1}}{r_{2}}\right) .
$$

We will need the following relation:

$$
\left\|\sqrt{P_{1}} A\right\|^{2}=-3 S_{3}=(n-1) S_{1} .
$$

The first equality is a general fact that follows from Proposition 2.1, part 3, by setting $r=1$ and $S_{2}=0$. The second equality is specific for Clifford tori with 
$S_{2}=0$ and can be proved as follows. Write:

$$
\begin{aligned}
S_{1}= & n_{1} \frac{r_{2}}{r_{1}}-n_{2} \frac{r_{1}}{r_{2}} \\
S_{2}= & \frac{n_{1}\left(n_{1}-1\right)}{2}\left(\frac{r_{2}}{r_{1}}\right)^{2}+\frac{n_{2}\left(n_{2}-1\right)}{2}\left(\frac{r_{1}}{r_{2}}\right)^{2}-n_{1} n_{2}, \\
S_{3}= & \frac{n_{1}\left(n_{1}-1\right)\left(n_{1}-2\right)}{6}\left(\frac{r_{2}}{r_{1}}\right)^{3}-\frac{n_{2}\left(n_{2}-1\right)\left(n_{2}-2\right)}{6}\left(\frac{r_{1}}{r_{2}}\right)^{3} \\
& +\frac{n_{1} n_{2}\left(n_{2}-1\right)}{2}\left(\frac{r_{1}}{r_{2}}\right)^{2} \frac{r_{2}}{r_{1}}-\frac{n_{1} n_{2}\left(n_{1}-1\right)}{2}\left(\frac{r_{2}}{r_{1}}\right)^{2} \frac{r_{1}}{r_{2}} .
\end{aligned}
$$

By introducing the condition $S_{2}=0$ into $S_{3}$, we obtain, after a long but straightforward computation, that

$$
3 S_{3}=\frac{1}{2}\left[-2(n-1) n_{1} \frac{r_{2}}{r_{1}}+2(n-1) n_{2} \frac{r_{1}}{r_{2}}\right]=-(n-1) S_{1},
$$

and this proves our claim. Thus the Jacobi operator reduces to

$$
\begin{aligned}
& \quad T_{1}(f)=L_{1}(f)+\left\{\left\|\sqrt{P_{1}} A\right\|^{2}+\operatorname{trace} P_{1}\right\} f=L_{1}(f)+2(n-1) S_{1} f . \\
& \text { If } \varphi=\text { const., } L_{1}(\varphi)=0 \text { and }
\end{aligned}
$$$$
T_{1}(\varphi)+2(n-1) S_{1} \varphi=0 .
$$

Thus the first eigenvalue of $T_{1}$ is negative, hence $\operatorname{Ind}(M)$ is at least 1 . Now let us look at the second eigenvalue of $T_{1}$. By using the expression of the eigenvalues of $P_{1}$ given above, we have that

$$
\begin{aligned}
L_{1}(f) & =\operatorname{div}\left(P_{1} \nabla f\right) \\
& =\left\{\left(n_{1}-1\right) \frac{r_{2}}{r_{1}}-n_{2} \frac{r_{1}}{r_{2}}\right\} \Delta^{n_{1}}(f)+\left\{n_{1} \frac{r_{2}}{r_{1}}-\left(n_{2}-1\right) \frac{r_{1}}{r_{2}}\right\} \Delta^{n_{2}}(f),
\end{aligned}
$$

where $\Delta^{n_{i}}$ is the Laplacian in $S^{n_{i}}\left(r_{i}\right), i=1,2$. Thus the second eigenvalue of $L_{1}$ is given by

$$
\lambda_{2}=-\left\{\left(n_{1}-1\right) \frac{r_{2}}{r_{1}}-n_{2} \frac{r_{1}}{r_{2}}\right\} \nu_{2}^{\Delta^{n_{1}}}+\left\{n_{1} \frac{r_{2}}{r_{1}}-\left(n_{2}-1\right) \frac{r_{1}}{r_{2}}\right\} \nu_{2}^{\Delta^{n_{2}}},
$$

where $\nu_{2}^{\Delta^{n_{i}}}$ is the first nonzero eigenvalue of $\Delta^{n_{i}}$ that corresponds to an eigenfunction which is invariant by the antipodal map (see [BGM] chap III, CII). Thus

$$
\begin{aligned}
\lambda_{2} & =-\left[\left\{\left(n_{1}-1\right) \frac{r_{2}}{r_{1}}-n_{2} \frac{r_{1}}{r_{2}}\right\} \frac{n_{1}}{r_{1}^{2}}+\left\{n_{1} \frac{r_{2}}{r_{1}}-\left(n_{2}-1\right) \frac{r_{1}}{r_{2}}\right\} \frac{n_{2}}{r_{2}^{2}}\right] \\
& =\frac{-1}{r_{1}^{3} r_{2}^{3}}\left\{\left[n_{1}\left(n_{1}-1\right)-n_{1}(n-1) r_{1}^{2}\right] r_{2}^{2}+\left[n_{2}(n-1) r_{2}^{2}-n_{2}\left(n_{2}-1\right)\right] r_{1}^{2}\right\} .
\end{aligned}
$$


Observe that

$$
S_{1}=n_{1} \frac{r_{2}}{r_{1}}-n_{2} \frac{r_{1}}{r_{2}}=\frac{n_{1} r_{2}^{2}-n_{2} r_{1}^{2}}{r_{1} r_{2}} .
$$

The fact that $S_{2}=0$ is equivalent to

$n(n-1) r_{1}^{4}-2 n_{1}(n-1) r_{1}^{2}+n_{1}\left(n_{1}-1\right)=n(n-1) r_{2}^{4}-2 n_{2}(n-1) r_{1} 2^{2}+n_{2}\left(n_{2}-1\right)=0$.

By using (7) and (8), we have that

$$
\left[n_{1}\left(n_{1}-1\right)-n_{1}(n-1) r_{1}^{2}\right] r_{2}^{2}=(n-1) S_{1} r_{1}^{3} r_{2}^{3}
$$

and

$$
\left[n_{2}(n-1) r_{2}^{2}-n_{2}\left(n_{2}-1\right)\right] r_{1}^{2}=(n-1) S_{1} r_{1}^{3} r_{2}^{3}
$$

Thus,

$$
\lambda_{2}=-2(n-1) S_{1} .
$$

Since the second eigenvalue of $T_{1}$ is given by $\lambda_{2}+2(n-1) S_{1}$, it is equal to zero. This shows then that the Clifford hypersurfaces of $\mathbb{P}(\mathbb{R})^{n+1}$ have index one.

Remark. Observe that, by equation (7), the condition $S_{1} \geq 0$ means that

$$
n_{1} r_{2}^{2}-n_{2} r_{1}^{2} \geq 0 \text {. }
$$

On the other hand, since $\beta=\left(\frac{r_{2}}{r_{1}}\right)^{2}$, the above inequality implies that

$$
n_{1} \beta \geq n_{2} .
$$

The condition $S_{2}=0$ is equivalent to

$$
n_{1}\left(n_{1}-1\right) \beta^{2}-2 n_{1} n_{2} \beta+n_{2}\left(n_{2}-1\right)=0,
$$

and one can easily see that only one solution of (10) is compatible with (9).

\section{A gap theorem for hypersurfaces of the sphere with $R=1$}

In this section we prove a gap theorem for hypersurfaces of the sphere with $R=1$.

Theorem 3.1 (Theorem 1 of the Introduction). Let $x: M^{n} \rightarrow S^{n+1}(1)$ be $a$ closed orientable hypersurface with scalar curvature $R=1$ (equivalently, $S_{2}=0$ ). Assume that $S_{1}$ does not change sign and choose the orientation such that $S_{1} \geq 0$. Assume further that

$$
\left\|\sqrt{P_{1}} A\right\|^{2} \leq \operatorname{trace} P_{1} .
$$

Then:

(i) $\left\|\sqrt{P_{1}} A\right\|^{2}=\operatorname{trace} P_{1}$. 
(ii) $M^{n}$ is either a totally geodesic submanifold or $M^{n}=S^{n_{1}}\left(r_{1}\right) \times S^{n_{2}}\left(r_{2}\right) \subset$ $S^{n+1}(1)$, where $n_{1}+n_{2}=n, r_{1}^{2}+r_{2}^{2}=1$ and $\left(\frac{r_{2}}{r_{1}}\right)^{2}=\beta$ satisfies the quadratic equation:

$$
n_{1}\left(n_{1}-1\right) \beta^{2}-2 n_{1} n_{2} \beta+n_{2}\left(n_{2}-1\right)=0 .
$$

Proof. Let us calculate $\frac{1}{2} L_{1}\|A\|^{2}$. Since $R=1, S_{2}=n(n-1)(R-1)=0$, by the Gauss' formula. Thus $\|A\|^{2}=(n H)^{2}=S_{1}^{2}$. Hence,

$$
\frac{1}{2} L_{1}\|A\|^{2}=\frac{1}{2} L_{1} S_{1}^{2}=S_{1} L_{1} S_{1}+\left\langle P_{1} \nabla S_{1}, \nabla S_{1}\right\rangle .
$$

From $[\mathrm{AdCC}]\left(\right.$ Lemma 3.7), by using that $2 S_{2}=n(n-1)(R-1)=0$, we have

$$
L_{1} S_{1}=|\nabla A|^{2}-\left|\nabla S_{1}\right|^{2}+n\|A\|^{2}-S_{1}^{2}+3 S_{1} S_{3} .
$$

Therefore,

$$
L_{1} S_{1}=|\nabla A|^{2}-\left|\nabla S_{1}\right|^{2}+(n-1) S_{1}^{2}+3 S_{1} S_{3} .
$$

Now, by using Proposition 2.1 (3), we obtain that

$$
\left\|\sqrt{P_{1}} A\right\|^{2}=\operatorname{trace} P_{1} A^{2}=-3 S_{3} .
$$

Then, equation (11) becomes

$$
L_{1} S_{1}=|\nabla A|^{2}-\left|\nabla S_{1}\right|^{2}+(n-1) S_{1}^{2}-S_{1}\left\|\sqrt{P_{1}} A\right\|^{2} .
$$

Thus,

$$
\begin{aligned}
\frac{1}{2} L_{1}\|A\|^{2} & =S_{1} L_{1} S_{1}+\left\langle P_{1} \nabla S_{1}, \nabla S_{1}\right\rangle \\
& =S_{1}\left(|\nabla A|^{2}-\left|\nabla S_{1}\right|^{2}+(n-1) S_{1}^{2}-3 S_{1}\left\|\sqrt{P_{1}} A\right\|^{2}\right)+\left\langle P_{1} \nabla S_{1}, \nabla S_{1}\right\rangle \\
& =S_{1}\left(|\nabla A|^{2}-\left|\nabla S_{1}\right|^{2}\right)+S_{1}^{2}\left((n-1) S_{1}-\left\|\sqrt{P_{1}} A\right\|^{2}\right)+\left\langle P_{1} \nabla S_{1}, \nabla S_{1}\right\rangle .
\end{aligned}
$$

Since $M$ is compact, we obtain

$$
\begin{gathered}
0=\frac{1}{2} \int_{M} L_{1}\|A\|^{2} d M \\
=\int_{M}\left\{S_{1}\left(|\nabla A|^{2}-\left|\nabla S_{1}\right|^{2}\right)+S_{1}^{2}\left((n-1) S_{1}-\left\|\sqrt{P_{1}} A\right\|^{2}\right)+\left\langle P_{1} \nabla S_{1}, \nabla S_{1}\right\rangle\right\} d M .
\end{gathered}
$$

We recall the following result (see $[\mathrm{AdCC}]$ - Lemma 4.1):

Lemma 3.1 ([AdCC]). Let $M$ be an $n$-dimensional compact hypersurface in an $(n+1)$-dimensional unit sphere $S^{n+1}$. If the normalized scalar curvature $R$ is constant and $R-1 \geq 0$, then

$$
|\nabla A|^{2}-\left|\nabla S_{1}\right|^{2} \geq 0 .
$$


Since $S_{1} \geq 0$ and $P_{1}$ is positive, we have that

$$
\left\langle P_{1} \nabla S_{1}, \nabla S_{1}\right\rangle=\left\|\sqrt{P_{1}} \nabla S_{1}\right\|^{2} \geq 0 .
$$

Our hypothesis and inequalities (13) and (14) implies that the right-hand side of (12) is non-negative. Thus we conclude that

$$
S_{1}\left(|\nabla A|^{2}-\left|\nabla S_{1}\right|^{2}\right)+S_{1}^{2}\left((n-1) S_{1}-\left\|\sqrt{P_{1}} A\right\|^{2}\right)+\left\langle P_{1} \nabla S_{1}, \nabla S_{1}\right\rangle=0 .
$$

Since each term in above equation is non-negative, we have

$$
S_{1}\left((n-1) S_{1}-\left\|\sqrt{P_{1}} A\right\|^{2}\right)=0 .
$$

Observe that when $S_{1}=0,\|A\|^{2}=0$ and $\left\|\sqrt{P_{1}} A\right\|^{2}=0$. Since by Lemma 2.1, trace $P_{1}=(n-1) S_{1}$, the first part of the theorem is proved.

Now, let us assume that $\left\|\sqrt{P_{1}} A(p)\right\|^{2}=(n-1) S_{1}(p)$, for all $p \in M$. If $S_{1}(p)=0$ for all $p \in M$, since $S_{2}=0,\|A\|^{2}=0$ and $M$ is totally geodesic. Let us suppose that there exists a point $p_{0}$ in $M$ such that $S_{1}\left(p_{0}\right)>0$. So the set $\mathcal{A} \subset M$ where $S_{1}(p)>0$ is an open and non-void set of $M$. We claim that $P_{1}$ is positive definite in $\mathcal{A}$. In fact, if $P_{1}$ has one eigenvalue equal to zero, then by Remark 2.1, $P_{1} A \equiv 0$ and since $\left\|\sqrt{P_{1}} A(p)\right\|^{2}=(n-1) S_{1}(p)$, we conclude that $S_{1}=0$, which is a contradiction. On each connected component of $\mathcal{A}$, we have that

$$
\left\langle P_{1} \nabla S_{1}, \nabla S_{1}\right\rangle=0
$$

and

$$
|\nabla A|^{2}-\left|\nabla S_{1}\right|^{2}=0
$$

Since $P_{1}$ is positive definite, the first equation implies that $\nabla S_{1}=0$. This implies that $|\nabla A|^{2}=0$, by the second equation, i.e., the second fundamental form of $M$ is covariant constant. It follows that the component $\mathcal{A}$ is a piece of a Clifford torus, by using the following theorem of H. B. Lawson ([L] - Theorem 4, see also [CdCK] Lemma 3).

Theorem $3.2[\mathrm{~L}]$. Let $M^{n}$ be an isometrically immersed hypersurface of $S^{n+1}$, over which the second fundamental form is covariant constant. Then, up to isometries of $S^{n+1}, M^{n}$ is an open set of $S^{k}(r) \times S^{n-k}\left(\sqrt{1-r^{2}}\right)$.

Finally, since along the boundary of $\mathcal{A},\|A\|^{2}=S_{1}^{2}=0$, we conclude that $\partial \mathcal{A}=\emptyset$ and $M$ is a Clifford torus.

\section{Characterization of index one closed hypersurfaces with $R=1$ in the real projective space}

In this section we will assume that the operator $L_{1}$ is elliptic and will describe the index of closed hypersurfaces in the real projective space $\mathbb{P}(\mathbb{R})^{n+1}$. In order to do that we are going to use the covering map of $S^{n+1}$ onto $\mathbb{P}(\mathbb{R})^{n+1}$. The following result will be needed. 
Lemma 4.1. Let $M^{n} \rightarrow S^{n+1}$ is a closed orientable hypersurface with $R=1$. Then the index of the quadratic form

$$
\begin{aligned}
I(f, f) & =-\int_{M} f T_{1} f d M \\
& =-\int_{M} f L_{1} f+\left((n-1) S_{1}-3 S_{3}\right) f^{2} d M
\end{aligned}
$$

is greater than one.

Proof. First of all observe that for constant functions $f=$ const., we have that

$$
\begin{aligned}
I(f, f) & =-\int_{M} f L_{1} f+\left((n-1) S_{1}-3 S_{3}\right) f^{2} d M \\
& =-\int_{M}\left((n-1) S_{1}-3 S_{3}\right) f^{2} d M<0 .
\end{aligned}
$$

Thus $\operatorname{ind}(M) \geq 1$.

Suppose that this index is equal to one. Let $\left\{e_{1}, \ldots, e_{n+2}\right\}$ be an orthonormal basis of $\mathbb{R}^{n+2}$. If we write the normal vector field of the immersion as $N=\sum_{i=1}^{n+2} n_{i} e_{i}$, we obtain that

$$
L_{1}\left(n_{i}\right)=3 S_{3} n_{i}, \text { for all } i=1, \ldots, n+2 \text {. }
$$

Thus

$$
I\left(n_{i}, n_{i}\right)=-\int_{M}\left((n-1) S_{1}\right) n_{i}^{2} d M \leq 0 .
$$

Since the functions $n_{i}$ are linearly independent, the index one hypothesis implies that $(n-1)$ of the $n_{i}^{\prime} s$ have to be null and since $|N|=1$, after reordering if necessary, we have $n_{1}=1$ and $n_{i}=0$ for $i=2, \ldots, n+2$. Thus the normal vector field $N=e_{1}$. This implies that $M^{n}$ is totally geodesic. On the other hand, since $L_{1}$ is elliptic, we have that $S_{1}>0$, and this contradicts the fact that $M^{n}$ is totally geodesic. We conclude then that $\operatorname{ind}(M)>1$.

The main result of this section is the following characterization of index one closed hypersurfaces of $\mathbb{P}(\mathbb{R})^{n+1}$.

Theorem 4.1 (Theorem 2 of the introduction). Let $x: M^{n} \rightarrow \mathbb{P}(\mathbb{R})^{n+1}(1)$ be $a$ closed two-sided hypersurface with scalar curvature one. Then $\operatorname{Ind}(M) \geq 1$ and if $\operatorname{Ind}(M)=1, M$ is the Clifford hypersurfaces obtained by the projection of the Clifford torus of Theorem 3.1.

Proof. The proof is inspired by the proof of the minimal case in [dCRR]. Observe that the index one hypothesis implies that $M$ must be connected. Since, by lemma $4.1, S^{n+1}$ does not have an index one hypersurface with $R=1, x$ cannot lift to an 
immersion of $M$ into $S^{n+1}$. Thus we obtain that there exists a connected twofold covering $\widetilde{M} \rightarrow M$ and an isometric immersion $\widetilde{x}: \widetilde{M} \rightarrow S^{n+1}$ which is locally congruent to the immersion of $M$ in $\mathbb{P}(\mathbb{R})^{n+1}$. An object in $\widetilde{M}$ that corresponds to an object in $M$ will be denoted by the same notation as in $M$. If we denote by $\pi: \widetilde{M} \rightarrow \widetilde{M}$ the isometric involution induced by the covering, then $\widetilde{x}$ must satisfy

$$
\widetilde{x} \circ \pi=-\widetilde{x}
$$

and, since $\widetilde{x}(M)$ is two-sided, $\widetilde{M}$ is orientable, and

$$
N \circ \pi=-N,
$$

where $N$ is the unit normal vector field of the immersion. We have that the immersion $\widetilde{x}$ is such that $R=1$ and $S_{3} \neq 0$. By ellipticity we can choose the orientation of $\widetilde{M}$ in such way that $S_{1}>0$.

Let $\lambda_{1}$ be the first eigenvalue of the operator

$$
T_{1}(\varphi)=L_{1}(\varphi)+\left((n-1) S_{1}+3 S_{3}\right) \varphi .
$$

We know that its first eigenspace is one-dimensional and generated by a function $\varphi$ that does not change sign on $\widetilde{M}$. Now, let $\varphi_{1}=\varphi \circ \pi$. Since $\pi$ is an isometry, we obtain that $T_{1}\left(\varphi_{1}\right)=\lambda_{1} \varphi_{1}$. This implies that $\varphi= \pm \varphi \circ \pi$. Observe that if $\varphi=-\varphi \circ \pi, \varphi$ has to change sign on $\widetilde{M}$. Thus $\varphi=\varphi \circ \pi$.

From the fact that $\operatorname{Ind}(M)=1$, we obtain that any function $u: \widetilde{M} \rightarrow \mathbb{R}$ such that $u \circ \pi=u$ and $\int_{\widetilde{M}} u \varphi d \widetilde{M}=0$ satisfies

$$
I(u, u)=-\int_{\widetilde{M}}\left\{u L_{1} u+\left((n-1) S_{1}+3 S_{3}\right) u^{2}\right\} d \widetilde{M} \geq 0 .
$$

Moreover, if such a function $u$ satisfies $I(u, u)=0$, then $u$ is a Jacobi function, that is,

$$
L_{1} u+\left((n-1) S_{1}+3 S_{3}\right) u=0 .
$$

Given $a, b \in \mathbb{R}^{n+2}$, let $\phi_{a, b}: \widetilde{M} \rightarrow \mathbb{R}^{n+2}$ be defined by

$$
\phi_{a, b}=\langle\widetilde{x}, a\rangle \widetilde{x}+\langle N, a\rangle N+\langle\widetilde{x}, b\rangle N .
$$

By doing the calculation coordinatewise and using equations (3) and (4) we have that

$$
L_{1}(\widetilde{x})=-(n-1) S_{1} \widetilde{x}
$$

and

$$
L_{1}(N)=3 S_{3} N
$$

Thus,

$$
\begin{gathered}
L_{1}(\langle\widetilde{x}, a\rangle \widetilde{x})=-2(n-1) S_{1}\langle\widetilde{x}, a\rangle \widetilde{x}-P_{1} A\left(a^{t}\right), \\
L_{1}(\langle N, a\rangle N)=6 S_{3}\langle N, a\rangle N-P_{1} A^{2}\left(a^{t}\right)
\end{gathered}
$$

and

$$
L_{1}(\langle\widetilde{x}, b\rangle N)=\left[-(n-1) S_{1}+3 S_{3}\right]\langle\widetilde{x}, b\rangle N-P_{1} A\left(b^{t}\right),
$$


where $a^{t}, b^{t}$ are the tangent projection of $a$ and $b$. This implies that

$$
T_{1}\left(\phi_{a, b}\right)=-\left[(n-1) S_{1}+3 S_{3}\right][\langle\widetilde{x}, a\rangle \widetilde{x}-\langle N, a\rangle N]+X_{a, b},
$$

where $X_{a, b}$ is a tangent vector field. Then,

$$
\begin{gathered}
-\int_{\widetilde{M}}\left\langle T_{1}\left(\phi_{a, b}\right), \phi_{a, b}\right\rangle d \widetilde{M} \\
=\int_{\widetilde{M}}\left[(n-1) S_{1}+3 S_{3}\right]\left[\langle\widetilde{x}, a\rangle^{2}-\langle N, a\rangle^{2}-\langle\widetilde{x}, b\rangle\langle N, a\rangle\right] d \widetilde{M} .
\end{gathered}
$$

Now, by (2), we have

$$
\begin{gathered}
\int_{\widetilde{M}}\left[(n-1) S_{1}+3 S_{3}\right]\langle\widetilde{x}, b\rangle\langle N, a\rangle d \widetilde{M} \\
=-\int_{\widetilde{M}}\left\{\langle N, a\rangle L_{1}(\langle\widetilde{x}, b\rangle)-\langle\widetilde{x}, b\rangle L_{1}(\langle N, a\rangle)\right\} d \widetilde{M}=0 .
\end{gathered}
$$

Thus,

$$
-\int_{\widetilde{M}}\left\langle T_{1}\left(\phi_{a, b}\right), \phi_{a, b}\right\rangle d \widetilde{M}=\int_{\widetilde{M}}\left[(n-1) S_{1}+3 S_{3}\right]\left[\langle\widetilde{x}, a\rangle^{2}-\langle N, a\rangle^{2}\right] d \widetilde{M} .
$$

Observe that the above expression does not depend on $b$. We are going to show that for any $a \in \mathbb{R}^{n+2}$, it is possible to choose $b \in \mathbb{R}^{n+2}$ such that $\int_{\widetilde{M}} \varphi \phi_{a, b} d \widetilde{M}=0$. To do this, consider a linear map $F: \mathbb{R}^{n+2} \rightarrow \mathbb{R}^{n+2}$ given by

$$
F(b)=\int_{\widetilde{M}} \varphi\langle\widetilde{x}, b\rangle N d \widetilde{M}
$$

We claim that $F$ is injective (thus a linear isomorphism). In fact, if $b \neq 0$ is such that $F(b)=0$, one has that (17), with $\phi=\phi_{0, b}=\langle\widetilde{x}, b\rangle N$, implies that

$$
I(\phi, \phi)=0 \text {. }
$$

Then, $T_{1}(\phi)=0$. On the other hand, for $a=0$,

$$
T_{1}(\phi)=X_{0, b}=-P_{1} A\left(b^{t}\right)=0,
$$

where $b^{t}$ is the tangent projection of $b$ along $\widetilde{M}$. Since $P_{1}$ is positive definite, (18) says that $A\left(b^{t}\right)=0$ on $\widetilde{M}$, which is the same that $\langle N, b\rangle$ is constant along $\widetilde{M}$. As we have that $N \circ \pi=-N$, we get that $\langle N, b\rangle=0$. This implies that the function $u=\langle\widetilde{x}, b\rangle$ satisfies that $\operatorname{Hess} u(X, Y)=\langle X, Y\rangle u$. We need the following result of M. Obata.

Theorem $4.2([\mathrm{O}]$ - Theorem A). In order that a complete Riemannian manifold of dimension $n \geq 2$ admit a non-constant function $\phi$ with $\operatorname{Hess} \phi(X, Y)=$ $c^{2} \phi\langle X, Y\rangle$, it is necessary and sufficient that the manifold be isometric to a sphere $S^{n}(c)$ of radius $\frac{1}{c}$ in the $(n+1)$ Euclidean space. 
Thus, if $u$ is non-constant, then $\widetilde{M}$ is isometric to a unit sphere and since $\widetilde{M}$ is isometrically immersed in $S^{n+1}(1)$, this implies that $\widetilde{M}$ is totally geodesic. On the other hand, if $u$ is constant, $\widetilde{M}$ is totally umbilic. Since $S_{2}=0, \widetilde{M}$ is again totally geodesic. In both cases, $S_{1}^{2}=|A|^{2}=0$, which is a contradiction to the fact that $S_{1}>0$. Thus the claim is proved.

Take an orthonormal basis $\left\{a_{1}, \ldots, a_{n+2}\right\}$ of $\mathbb{R}^{n+2}$. By using the isomorphism $F$, for any $i=1, \ldots, n+2$, it is possible to find $b_{i} \in \mathbb{R}^{n+2}$ such that $\int_{\widetilde{M}} \varphi \phi_{a_{i}, b_{i}} d \widetilde{M}=0$. Thus each coordinate $\phi_{i j}$ of $\phi_{a_{i}, b_{i}}$ is such that $\int_{\widetilde{M}} \varphi \phi_{i j} d \widetilde{M}=0$. Then, $I\left(\phi_{i j}, \phi_{i j}\right) \geq 0$. From equation (17), we have

$$
\begin{aligned}
0 & \leq \sum_{i=1}^{n+2} \int_{\widetilde{M}}\left[(n-1) S_{1}+3 S_{3}\right]\left[\left\langle\widetilde{x}, a_{i}\right\rangle^{2}-\left\langle N, a_{i}\right\rangle^{2}\right] d \widetilde{M} \\
& =\sum_{i=1}^{n+2} \int_{\widetilde{M}}\left[(n-1) S_{1}+3 S_{3}\right]\left(|\widetilde{x}|^{2}-|N|^{2}\right) d \widetilde{M}=0 .
\end{aligned}
$$

This implies that $T_{1}\left(\phi_{a_{i}, b_{i}}\right)=0, i=1, \ldots, n+2$. Hence, $\left\langle T_{1}\left(\phi_{a_{i}, b_{i}}\right), \widetilde{x}\right\rangle=0$ and, by equation (16), we obtain that

$$
\left[(n-1) S_{1}+3 S_{3}\right]\left\langle\widetilde{x}, a_{i}\right\rangle=0, i=1, \ldots, n+2 .
$$

But this is only possible if $(n-1) S_{1}+3 S_{3}=0$. Since $\left\|\sqrt{P_{1}} A\right\|^{2}=-3 S_{3}=(n-1) S_{1}$, theorem (3) implies that $\widetilde{M}$ is a Clifford torus.

\section{References}

[AdCC] H. Alencar, M. do Carmo and A. Colares, Stable hypersurfaces with constant scalar curvature, Math. Z. 213 (1993), 117-131.

[BC] J. L. Barbosa and A. Colares, Stability of hypersurfaces with constant $r$-mean curvature, Annals of Global Analysis and Geometry 15 (1997), 277-297.

[BGM] M. Berger, P. Gauduchon et E. Mazet, Le spectre d'une variété Riemanniene, Lecture Notes in Math. 194, Springer-Verlag, Berlin-New York, 1971.

[CdCK] S. S. Chern, M. do Carmo and S. Kobayashi, Minimal submanifolds of a sphere with second fundamental form of constant length, Functional Analysis and Related Fields, edited by Felix E. Browder, 1970, 59-75.

[dCRR] M. do Carmo, M. Ritoré and A. Ros, Compact minimal hypersurfaces with index one in the real projective space, Comment. Math. Helv. 75, no. 2 (2000), 247-254.

[HL] J. Hounie and M. L. Leite, Two-ended hypersurfaces with zero scalar curvature, Indiana Univ. Math. J. 48 (1999), 867-882.

[L] H. B. Lawson, Local rigidity theorems for minimal hypersurfaces, Ann. of Math. (2) 89 (1969), 187-197.

[M] H. F. Münzner, Isoparametriche hyperflächen in sphären I, Math. Ann. 215, no. 1 (1980), 57-71.

[O] M. Obata, Certain conditions for a Riemannian manifold to be isometric with a sphere, J. Math. Soc. Japan 14, no. 3 (1962), 333-340. 
[Re] R. C. Reilly, Variational properties of functions of the mean curvatures for hypersurfaces in space forms, J. Diff. Geom. 8 (1973), 465-477.

[Ro] H. Rosenberg, Hypersurfaces of constant curvature in space forms, Bull. Sc. Math., $2^{a}$ série 117 (1993), 211-239.

[S] J. Simons, Minimal varieties in riemannian manifolds, Ann. of Math. (2) 88 (1968), $62-105$.

Hilário Alencar

Universidade Federal de Alagoas

Departamento de Matemática

57072-900, Maceió - AL

Brazil

e-mail: hilario@mat.ufal.br

Walcy Santos

Universidade Federal do Rio de Janeiro

Departamento de Matemática

Caixa Postal 68530

21945-970 Rio de Janeiro - RJ

Brazil

e-mail: walcy@im.ufrj.br

(Received: October 12, 2001)

\section{Manfredo do Carmo}

Instituto de Matemática Pura e Aplicada (IMPA)

Estrada Dona Castorina 110

22460-320, Rio de Janeiro - RJ

Brazil

e-mail: manfredo@impa.br.

To access this journal online:

(20) http://www.birkhauser.ch 